Published in final edited form as:

Biotechnol Bioeng. 2014 March ; 111(3): 441-453. doi:10.1002/bit.25160.

\title{
Nanocomposite hydrogels for biomedical applications
}

\author{
Akhilesh K. Gaharwar ${ }^{1}$, Nicholas A. Peppas ${ }^{2}$, and Ali Khademhosseini ${ }^{3,4,5}$ \\ Ali Khademhosseini: alik@rics.bwh.harvard.edu \\ ${ }^{1}$ Department of Biomedical Engineering, Texas A \& M University, College Station, Texas 77843 \\ ${ }^{2}$ Departments of Chemical Engineering, Biomedical Engineering and Pharmacy, The University of \\ Texas at Austin, Austin, Texas 78712 \\ ${ }^{3}$ Wyss Institute for Biologically Inspired Engineering, Harvard University, Boston, Massachusetts \\ 02115 \\ ${ }^{4}$ Department of Medicine, Center for Biomedical Engineering, Brigham and Women's Hospital, \\ Harvard Medical School, Cambridge, Massachusetts 02139 \\ ${ }^{5}$ Harvard-MIT Division of Health Sciences and Technology, Massachusetts Institute of \\ Technology, Cambridge, Massachusetts 02139
}

\section{Abstract}

\begin{abstract}
Hydrogels mimic native tissue microenvironment due to their porous and hydrated molecular structure. An emerging approach to reinforce polymeric hydrogels and to include multiple functionalities focuses on incorporating nanoparticles within the hydrogel network. A wide range of nanoparticles, such as carbon-based, polymeric, ceramic, and metallic nanomaterials can be integrated within the hydrogel networks to obtain nanocomposites with superior properties and tailored functionality. Nanocomposite hydrogels can be engineered to possess superior physical, chemical, electrical, and biological properties. This review focuses on the most recent developments in the field of nanocomposite hydrogels with emphasis on biomedical and pharmaceutical applications. In particular, we discuss synthesis and fabrication of nanocomposite hydrogels, examine their current limitations and conclude with future directions in designing more advanced nanocomposite hydrogels for biomedical and biotechnological applications.
\end{abstract}

\section{Keywords}

nanocomposite hydrogels; nanoparticles; biomedical applications; tissue engineering; drug delivery

\section{Introduction}

Engineering complex tissues that can mimic or stimulate native tissue functions hold enormous promise in treating organ failures resulting from injuries, aging, and diseases (Khademhosseini et al., 2009; Langer and Vacanti, 1993; Peppas et al., 2006). The inability to mimic the complex tissue architecture, and to provide the essential cellular microenvironment are some of the challenges that need to be addressed to control the formation of functional tissues. Designing advanced biomaterials with controlled physical, chemical, electrical, and biological properties will therefore be beneficial to facilitate the

(C) 2013 Wiley Periodicals, Inc.

Correspondence to: Nicholas A. Peppas; Ali Khademhosseini, alik@rics . bwh . harvard. edu. 
formation of functional tissues (Fisher et al., 2010; Lowman and Peppas, 1999; Slaughter et al., 2009).

Among different biomaterials, hydrogels are one of the potential candidates as they can mimic the physical, chemical, electrical, and biological properties of most biological tissues (Fisher et al., 2010; Kloxin et al., 2010; Slaughter et al., 2009). Hydrogels possess a threedimensional (3D) highly hydrated polymeric network, and can hold up to 20- to 40-fold more water compared with their dry weight. Due to their unique physical properties, these networks can be shaped or casted into various sizes and shapes.

Over the last decade, new applications of hydrogels have emerged, particularly in stem cell engineering, immunomodulation, cellular and molecular therapies, and cancer research (Discher et al., 2009; Tibbitt and Anseth, 2009). Most of these applications demand multiple functionalities of the hydrogel network and dynamic interactions between the surrounding matrices and the cells (Tibbitt and Anseth, 2009). A range of innovations, in polymer chemistry, micro-and nanofabrication technologies, and biomolecular engineering, have pushed the limit for designing composite hydrogel networks with customized functionality (Annabi et al., 2014). Recent trends also indicate significant and growing interest in developing nanocomposite hydrogels for various biomedical applications (Fig. 1). Nanocomposite hydrogels, also known as hybrid hydrogels, can be defined as hydrated polymeric networks, either physically or covalently crosslinked with each other and/or with nanoparticles or nanostructures. Although there are various opportunities for nanocomposite biomaterials, this review will specifically focus on biomedical applications specifically in regenerative medicine, drug delivery, biosensors, and bioactuators.

A range of nanoparticles such as carbon-based nanomaterials (carbon nanotubes (CNTs), graphene, nano diamonds), polymeric nanoparticles (polymer nanoparticles, dendrimers, hyperbranched polyesters), inorganic/ceramic nanoparticles (hydroxyapatite, silica, silicates, calcium phosphate), and metal/metal-oxide nanoparticles (gold, silver, iron-oxide) are combined with the polymeric network to obtain nanocomposite hydrogels (Fig. 2). These nanoparticles physically or covalently interact with the polymeric chains, and result in novel properties of the nanocomposite network (Goenka et al., 2014; Schexnailder and Schmidt, 2009; Thomas et al., 2006). Developing nanocomposite hydrogels with tailored functionality has opened up new possibilities in developing advanced biomaterials for various biomedical and biotechnological applications (Lowman et al., 2004; Peppas et al., 2007).

In this review, we focused on the recent advances in the designing and developing nanocomposite hydrogels with tailored physical properties and custom functionality. We will discuss the different types of nanocomposite hydrogels and highlight some of the emerging biomedical and biotechnological applications of these hydrogels. In particular, the emerging trends in designing complex hydrogels with multiple functionalities are discussed. We will also evaluate the nanocomposite hydrogels based on the two-phase and multi-phase systems. Additionally, some of the most critical challenges associated with the design, fabrication, and evaluation of nanocomposite hydrogels are highlighted.

\section{Nanocomposite Hydrogels From Carbon-Based Nanomaterials}

Carbon-based nanomaterials such as CNTs, graphene, buckminsterfullerene (C60), and nanodiamonds are being investigated for potential applications in biomedicine (Cha et al., 2013; Goenka et al., 2014). Specifically, CNTs and graphene are extensively used to incorporate multifunctionality such as high mechanical, electrical conductivity, and optical properties to the synthetic or natural polymers (Cha et al., 2013). For example, both CNTsor graphene-based nanocomposite hydrogels are evaluated for applications such as actuators, 
conductive tapes, biosensors, tissue engineering scaffolds, drug delivery systems, and biomedical devices (Goenka et al., 2014; Kuilla et al., 2010).

CNTs are classified as multi-wall or single-wall CNTs. Both the types of CNTs are investigated for biomedical and biotechnological applications, however, one of the problems associated with CNTs is their hydrophobic nature that results in limited interaction with hydrophilic polymers. This is mainly attributed to the strong $\pi-\pi$ interactions between the CNTs, which results in the formation of aggregates. To circumvent this drawback, several techniques are developed to enhance the dispersion of the CNTs within the nanocomposite network. For example, CNT surfaces are modified using various polar groups such as amines $\left(\mathrm{NH}_{2}\right)$, hydroxyls $(\mathrm{OH})$, and carboxyls $(\mathrm{COOH})$ to facilitate their dispersion in hydrophilic polymers (Ma et al., 2010). Other strategies implemented to improve the solubility of the CNTs in aqueous solutions include the use of single-stranded DNA (ssDNA), proteins, and surfactants to modify the surface properties.

To engineer complex tissues, hydrogel networks with tailored functionality should interact with cells and direct the formation of functional tissue. To mimic complex tissue structures, a plethora of new approaches to design customized nanocomposite hydrogels are emerging (Schexnailder and Schmidt, 2009; Cha et al., 2013; Goenka et al., 2014). Due to their high electrical conductivity, nanocomposites reinforced with CNTs can be used to engineer a range of electrically conductive tissues such as nerve, muscle, and cardiac tissues. In a preliminary study, methacrylated gelatin (GelMA) was reinforced with multiwall $\mathrm{COOH}-$ functionalized CNTs to fabricate hybrid nanocomposite hydrogels (Shin et al., 2011). Multiwall CNTs were coated with GelMA to generate a fibrous structure in which CNTs were imbedded within an interconnected and porous hydrogel network (Fig. 3). Almost threefold increase in the tensile modulus was observed due to the addition of $0.5 \%$ CNTs to the GelMA hydrogels. The increase in the mechanical strength of GelMA hydrogels, due to the addition of the multiwall CNTs, was attributed to the formation of a nanofibrous mesh-like network of GelMA-coated multiwall CNTs (Shin et al., 2011). It was demonstrated that cardiomyocytes seeded onto CNT-GelMA nanocomposite hydrogels had a threefold increase in their spontaneous beating frequency when compared with cells that were seeded on GelMA-alone hydrogels (Fig. 3; Shin et al., 2013). In addition, cardiomyocytes seeded onto the CNT-GelMA hydrogels showed highly aligned sarcomeric structures with tight intercellular junctions. Interestingly, the external voltage required to induce beating in the cells was significantly decreased due to the addition of the CNTs. This was mainly attributed to the higher electrical conductivity of the CNTs and the formation of a conductive network within the nanocomposite hydrogels. Engineered cardiac tissues when released from the substrate, spontaneously actuated and moved within a fluid environment due to the cyclical contraction of the cells. Although CNT-based hydrogels are able to demonstrate that labgrown tissues can mimic some of the function of the native tissue, their utility in the body as tissue replacements needs to be further investigated due to their cytotoxicity concerns.

Another approach to functionalize the surface of carbon-based nanomaterials is to graft polymer chains onto the CNT surfaces. In this approach, the CNTsurface is shielded and the surrounding polymeric network mainly perceives the surface grafted polymer (Liu and Guo, 2010). This shielding approach can be used to enhance the dispersion of CNTs and physical interactions between CNTs and polymer. For example, the surface of the multiwall CNTs was hybridized with amyloid fibrils to generate fibrous hydrogels (Li and Mezzenga, 2012). A significant decrease in the gelling concentration of the amyloid fibrils was observed due to the addition of the CNTs. It was observed that grafting the amyloid fibers on the CNTsurfaces significantly enhanced the polymer-nanotube interactions. This approach can be extended to various protein-CNT systems as many proteins can form amyloid fibers. It is 
envisioned that the protein-CNTs nanocomposite hydrogels can be used to design responsive hydrogels for applications such as tissue engineering, drug delivery, and biosensing.

Graphene is a two dimensional carbon-based nanomaterial with high mechanical strength and is an excellent conductor of heat and electricity. To enhance the solubility of graphene in physiological conditions, graphene sheets can be treated with strong oxidizers to obtain graphite oxide (GO). GO can interacts with hydrophilic polymers to form physically or covalently crosslinked networks. In one study, genetically engineered elastin-like polypeptides (ELPs) were non-covalently reinforced with GO to obtain hydrogel actuators that were stimuli responsive (Wang et al., 2013). The resulting hybrid GO-ELPs hydrogels could be mechanically actuated to bend, stretch and twist when subjected to different radiation intensities.

Despite the improvement in the physical properties of the polymeric networks due to the addition of functionalized nanoparticles, in most studies nanoparticles were used as physical fillers in the polymer networks, and were not covalently incorporated into the resulting materials. The covalent crosslinking between polymer and nanoparticles enables the transfer of mechanical force within the cross-linked network, resulting in enhanced mechanical strength and toughness. For example, the molecular dynamic simulation indicates that by covalently conjugating the polymer chains with the CNTs, an order of magnitude increase in the mechanical strength was expected by involving less than $1 \%$ of the surface atoms present on the nanoparticle (Frankland et al., 2002). Thus, carbon based nanoparticles such as CNTs can be used as a crosslinking agent if they can be chemically conjugated to the polymeric network.

In a recent study, mechanically stiff and highly resilient nanocomposite hydrogels were fabricated by covalently conjugating GO sheets to polyacrylamide (Liu et al., 2012). In this study, GO sheets were functionalized via radiation-induced peroxidation to obtain graphene peroxide (GPO). The surface functionalization of GO enhanced the interfacial interactions between the GPO and the polymeric chains. The functionalized GPO was covalently crosslinked with polyacrylamide to obtain nanocomposite hydrogels. The chemical crosslinking between the polymer chains and GO sheets resulted in a mechanically stiff and elastomeric hydrogels. For example, more than $900 \%$ and $500 \%$ increases in tensile strength and elongation, respectively, were observed due to the addition of the GPO $(3 \mathrm{mg} / \mathrm{mL})$ compared with conventional polymeric hydrogels. This approach can be used to fabricate elastomeric scaffolds for tissues that are under constant mechanical stress and electrical stimulation. Although the functionalization of GO enhances the physical and chemical stability of the crosslinked network, additional studies are needed to investigate the interactions between mammalian cells and these hybrid nanocomposite networks.

Overall, during the past decade significant efforts have been focused on designing and developing hybrid hydrogels containing carbon-based nanoparticles (specifically CNTs and GO) for biomedical applications. Mechanically tough and electrically conductive hydrogels are developed by reinforcing the polymeric network by CNTs or GO. These nanocomposite hydrogels combine multiple functionalities, which is otherwise not possible by using conventional polymers. However, more detailed and mechanistic studies are required to investigate the biological properties of such hydrogels under in vitro and in vivo conditions.

\section{Nanocomposite Hydrogels From Polymeric Nanoparticles}

Polymeric nanoparticles such as dendrimers, hyperbranched polymers, liposomes, polymeric micells, nanogels, and core-shell polymeric particles have gained great attention in drug delivery application due to their ability to entrap hydrophobic or hydrophilic drugs (Joshi 
and Grinstaff, 2008; Seidlits and Peppas, 2007). Among them, dendrimers and hyperbranched polymeric nanoparticles are attractive because of their highly branched and spherical structure. Due to their unique nanostructure, they display a multitude of functional groups on their periphery, resulting in higher reactivity and loading efficiency compared to the polymeric hydrogels fabricated from linear polymers (Gillies and Frechet, 2005). Additionally, a multitude of bioactive agents such as drugs, proteins, and genes can be encapsulated within highly branched structure or can be conjugated on the periphery of the nanoparticles.

Dendritic nanoparticles can be used to reinforce the hydrogel network via covalent or noncovalent interactions with the polymeric chains. For example, polyamidoamine (PAMAM) dendritic nanoparticles were physically integrated within collagen scaffolds to improve the structural integrity and mechanical stiffness (Zhong and Yung, 2009). Interestingly, the addition of PAMAM nanoparticles significantly improved human conjunctival fibroblast proliferation in collagen hydrogels. The enhanced cellular proliferation is attributed due to the increase in the stiffness of nanocomposite network due to the addition of nanoparticles. The applications of these hybrid hydrogels from dendrimers and hyperbranched nanoparticles are envisioned in pharmaceutical and biomedical area that requires porous structure with controlled drug release properties (Oral and Peppas, 2004; Peppas et al., 2000; Seidlits and Peppas, 2007).

Similarly, a tri-block copolymer made from poly(glycerol-succinic acid) dendrimers as terminal blocks and PEG as the linear core was evaluated for soft tissue engineering applications (Sontjens et al., 2006). By changing the dendrimer concentration, the stiffness of the hydrogels, the degradation properties and the hydration kinetics can be tailored. The nanocomposite hydrogels showed high stress-absorbing capacity and thus may be useful for cartilage tissue engineering applications. Interestingly, the encapsulated chondrocytes retained a rounded morphology and showed a significant increase in production of type II collagen and proteoglycans, due to the incorporation of the nanoparticles.

Most of the dendrimers/hyperbranched nanoparticles used to reinforce polymeric network for tissue engineering applications belong to the low generation macromolecules (2-3 generations), and therefore, hydrogels made from these nanoparticles possess poor drug loading efficiency and limited control over the release kinetic. To overcome these difficulties, hydrogels from hyperbranched poly(amine-ester) (HPE) nanoparticles (generation 5) were fabricated (Fig. 4) (Zhang et al., 2013). The periphery of the HPE nanoparticles was modified with photocrosslinkable moieties to form a covalently crosslinked network upon UV exposure. By controlling the crosslinking density, the physical properties of the resulting hydrogels including the microstructure and mechanical strength were tuned. These photocrosslinkable hydrogels are able encapsulate hydrophobic drug within the inner cavities of the nanoparticles. A controlled drug release from the nanocomposite network was observed for more than a week, which is otherwise difficult to achieve when using conventional hydrogels made from linear polymers.

Overall, a range of dendrimers/hyperbranched-based nanocomposite hydrogels with tailored physical and chemical properties has been evaluated for various tissue engineering and drug delivery applications. Mechanically stiff hydrogels with controlled drug delivery characteristics can be fabricated by combining dendrimers/hyperbranched nanoparticles with linear polymers. By providing appropriate therapeutic cues to the cells, a range of biological properties can also be controlled. However, most of the studies on dendrimers/ hyperbranched nanoparticles-based hydrogels have been performed in vitro and thus additional animal experiments are required to examine their behavior in vivo. 


\section{Nanocomposite Hydrogels From Inorganic Nanoparticles}

Inspired from the bioactive nanomaterials found in biological tissues, researchers are developing the next generation of advanced biomaterials by combining inorganic ceramic nanoparticles with natural or synthetic polymers. In the last few decades, a range of bioactive nanoparticles have been reported including hydroxyapatite (nHA), synthetic silicate nanoparticles, bioactive glasses, silica, calcium phosphate, glass ceramic, and $\beta$ wollastonite for biomedical applications (Hench and Polak, 2002).

Most of these inorganic nanoparticles consist of minerals that are already present in the body and are necessary for the normal functioning of human tissues and have shown favorable biological responses (Hoppe et al., 2011). For example, calcium is an important component of bone and plays a vital role in bone development and maintenance. The presence of intracellular calcium and phosphate in the osteoblasts promote the deposition of mineralized matrix and prevent bone loss. Similarly, silicon, another mineral component present in most of the ceramic nanoparticles, plays a key role in skeletal development. Silicon stimulates the osteogenic differentiation in human stem cells and also promotes collagen type I synthesis. Thus, incorporating such inorganic nanoparticles within polymeric hydrogels is expected to introduce bioactive characteristics to the network.

Various nanocomposite hydrogels have been synthesized by incorporating ceramic nanoparticles within the polymer matrix. For example, nHA was incorporated within poly(ethylene glycol) (PEG) matrix to obtain highly elastomeric nanocomposite hydrogels (Gaharwar et al., 2011a). Interestingly, the addition of nHA to the polymeric network imparted elastomeric properties, enhanced mechanical strength and improved the physiological stability of the nanocomposite networks. Although the exact interactions between $\mathrm{nHA}$ and polymeric network were not investigated, it was speculated that apart from physical, electrostatic, and ionic interactions were also playing an important role for the high toughness and elastomeric properties of the nanocomposite networks. Moreover, the addition of nHA resulted in enhanced cell adhesion characteristics when compared with PEG hydrogels. Similar results were obtained when nHA was replaced with silica nanospheres (Gaharwar et al., 2013b). The addition of silica nanoparticles to PEG hydrogels resulted in enhanced mechanical properties and cell adhesion characteristics. Due to enhanced bioactivity and high mechanical strength, these nanocomposite networks (PEGnHA and PEG-Silica) can be used as injectable fillers for orthopedic applications (Gaharwar et al., 2011a; Gaharwar et al., 2011b).

Synthetic silicates nanoparticles, also known as nanoclays, have been observed to improve the physical and mechanical properties of polymeric hydrogels (Bordes et al., 2009; Wu et al., 2010; Gaharwar et al., 2010, 2012). This is due to the anisotropic and plate-like, high aspect-ratio morphology of the nanoclay, resulting in high surface interactions between the polymers and the nanoparticles. Nanoclays are widely used to reinforce thermoplastic polymers in order to obtain hybrid structures with hierarchical structure, elastomeric properties, ultrastrong and stiff films, super gas-barrier membranes, superoleophobic surfaces, flame-retardant structures, and self-healing characteristic (Bordes et al., 2009; Wu et al., 2010). Despite their unique physical and chemical properties, only a few reports have evaluated nanoclay-based nanocomposite hydrogels for biomedical applications such as controlled cell adhesion surfaces, injectable drug delivery matrix, and antimicrobial films (Gaharwar et al., 2010, 2012; Dawson and Oreffo, 2013).

Recent studies have shown that nanoclay (synthetic silicate nanoplatelets) can induce osteogenic differentiation of hMSCs without the use of exogenous growth factors (Gaharwar et al., 2013a). These synthetic silicates trigger a series of events that follow the temporal 
pattern of osteogenic differentiation (alkaline phosphates (ALP)/RUNX2 transcripts upregulation, bone related matrix protein deposition (osteocalcin $(\mathrm{OCN})$ and osteopontin $(\mathrm{OPN})$ ), followed by matrix mineralization). These unique bioactive properties of the synthetic silicates may be processed to create devices such as injectable tissue repair matrices, bioactive fillers or therapeutic agents for triggering specific cellular responses towards bone-related tissue engineering approaches.

Synthetic silicate nanoplatelets, when mixed with linear and branched polymers, result in mechanically strong and tissue-adhesive nanocomposite hydrogels (Gaharwar et al., 2010, 2012; Schexnailder et al., 2010). The unique physical and chemical properties of silicatebased nanocomposites are attributed to the high surface interactions of polymer and silicate nanoparticles. Specifically, this behavior was mainly attributed to the non-covalent interactions, as the polymer chains reversibly adsorb and desorb on the silicate surfaces (Gaharwar et al., 2012). Such system show shear thinning characteristics and could therefore be used for minimally invasive therapies. In another study, the addition of silicates improved the elongation of the polymeric hydrogels due to the formation of physically crosslinked networks (Gaharwar et al., 2011b, c). Interestingly, silicates that were incorporated within the PEG network enhanced cell and tissue adhesive properties of the nanocomposite hydrogels (Fig. 5). This may be due to the protein adsorption on the surface of silicate particles that subsequently promotes cellular adhesion. Although preliminary investigation of synthetic silicate with mammalian cells suggests high cytocompatibility, additional in vivo experiments should be performed to examine the long-term cytotoxicity of silicate nanoparticles.

Other types of ceramic nanoparticles, such as calcium phosphate, bioglasses, and $\beta$ wollastonite, are also incorporated in different synthetic and natural polymers to obtain bioactive nanocomposite hydrogels (Schexnailder and Schmidt, 2009). Due to the high mechanical strength of these ceramic nanoparticles, they are mainly used to reinforce the polymeric network. The degradation or dissolution products of these ceramic nanoparticles can result in favorable biological response and thus providing opportunities for their use in various biomedical applications.

\section{Nanocomposite Hydrogels From Metal and Metal-Oxide Nanoparticles}

Various types of metallic nanoparticles used to fabricate nanocomposite hydrogels for biomedical applications include gold $(\mathrm{Au})$, silver $(\mathrm{Ag})$, and other noble metal nanoparticles, whereas metal-oxide nanoparticles include iron oxide $\left(\mathrm{Fe}_{3} \mathrm{O}_{4}, \mathrm{Fe}_{2} \mathrm{O}_{3}\right)$, titania $\left(\mathrm{TiO}_{2}\right)$, alumina, and zirconia (Schexnailder and Schmidt, 2009). Metal and metal-oxide nanoparticles have been shown to possess desired physical properties such as electrical conductivity (Au nanorods), magnetic properties (iron oxides), and antimicrobial properties (Ag nanoparticles). Thus, nanocomposite hydrogels containing metal or metal-oxide nanoparticles are extensively used as imaging agents, drug delivery systems, conductive scaffolds, switchable electronics, actuators, and sensors (Schexnailder and Schmidt, 2009).

The interactions between the polymer and the metal/metal-oxide nanoparticles are often weak. However, by functionalizing the nanoparticle surfaces, the interactions between the polymer and the nanoparticles can be enhanced (Balazs et al., 2006; Caruso, 2001). Such enhanced interactions significantly influence the physical, chemical, and biological properties of the nanocomposite hydrogels. For example, Au nanoparticles entrapped within the polymeric network consisting of hyaluronic acid (HA) and gelatin do not improve the mechanical properties (Skardal et al., 2010). However, when thiol-functionalized Au nanoparticles are allowed to crosslink with the polymeric network, significant increase in the stiffness are observed. Such augmentation in the physical properties is related to the 
enhanced interaction of nanoparticles with the polymer chains resulting in the formation of a crosslinked network.

Metallic nanoparticles possess superior electrical and thermal conductivity. When such conductive nanoparticles are embedded or entrapped within a polymeric hydrogel network, they enhance the electrical conductivity of the nanocomposite hydrogels. In a recent attempt, researchers incorporated $\mathrm{Au}$ nanowires within macroporous alginate hydrogels to enhance the electrical conductivity of the polymeric network (Dvir et al., 2011). Such conductive scaffolds can be used to engineer tissues that require propagation of electrical signals to facilitate the formation of functional tissues (Fig. 6). For example, neonatal rat cardiomyocytes when seeded within the conductive hydrogel network, contract synchronously when electrically stimulated (Dvir et al., 2011). Moreover, the addition of Au nanowire to the alginate hydrogel also showed a higher expression of the cardiac markers (such as troponin I and connexin 43) in the seeded cardiac cells when compared to the alginate hydrogels.

Application of nanocomposite hydrogels for biomedical biomedicine depends on the type of physical and chemical biomedicine of the network. For example, by incorporating magnetic nanoparticles within hydrogel network, the nanocomposite network can remotely interact with external magnetic fields. In a recent study, magnetic nanoparticles are covalently conjugated with biocompatible and thermoresponsive hydroxypropyl cellulose to obtain stimuli-responsive hydrogels (Gaharwar et al., 2009). When these hybrid hydrogels are subjected to external magnetic fields, the magnetic nanoparticle entrapped within the hydrogel network generate heat. The increase in the temperature of surrounding matrix above the lower critical solution temperature of the polymer, results in a coil-to-globule transition of the polymer chains. Such type of triggers can be used to release therapeutic agents or cells from the nanocomposite hydrogels.

Other types of metal-oxide nanoparticles can be used to enhance the bioactivity of hydrogels. For example, nanophase alumina and titania, when incorporated within polymeric matrix such as poly-(L-lactic-co-glycolic acid) (PLGA), result in enhanced osteoblast adhesion and proliferation (Price et al., 2003; Webster et al., 2001). However, very limited studies focus on using these nanoparticles to fabricate hydrogel structures. This is mainly attributed to limited interactions between nanoparticles (such as alumina and titania) with polymeric chains that does not result in any increase in physical properties of the hydrogel network. In a recent study, the surface of titania was functionalized with amine groups to facilitate covalent interactions between the nanoparticles and carboxymethylcellulose (Pasqui et al., 2011). These hybrid nanocomposite hydrogels can be used to encapsulate cells for tissue engineering applications.

Overall, various types metallic and metal-oxide nanoparticles are explored to design nanocomposite hydrogels with desired functionality. Besides using the metallic or metaloxide based nanocomposite hydrogels for tissue engineering applications, they are also explored for biosensing, diagnostic, and bioactuation applications.

\section{Next Generation of Nanocomposite Hydrogels}

Nanocomposite hydrogels are being increasingly evaluated for various biomedical applications. The enhanced surface interactions between the nanoparticles and the polymer chains result in material properties that may be useful for various biomedical applications. However, most of the current nanocomposite approaches lack control over some essential features such as stimuli responsiveness and biodegradation. To address these challenges, alternate strategies have been developed to design nanocomposite hydrogels with multiple functionalities. 
By combining multiple phases within a nanocomposite hydrogel network, several properties can be incorporated to mimic structure and properties of native tissues. For example, aliphatic ester dendrimers conjugated with PEG spacer can physically interact with inorganic nanoplatelets (ceramic nanoparticles) to form physically crosslinked hydrogels (Wang et al., 2010). Interestingly, the crosslinked networks has self-healing characteristics, high mechanical strength, and adhesive properties. This provides an example of a multicomponent system to obtain distinct functionality within a hybrid hydrogel network.

In another study, biomimetic adhesive nanocomposite hydrogels were fabricated by combining polyacrylamide (PAAm), silicate nanoplatelets, and dopamine methacrylamide (DMA) (Skelton et al., 2013). It was observed that the addition of DMA was necessary to enhance the stiffness and energy dissipation capability to mimic the properties of elastomeric tissues. The chemically crosslinked network and presence of DMA enhanced interfacial interactions of silicate and PAAm. Although, this study indicated that multicomponent hydrogels (PAAm-silicate-DMA) have superior physical and chemical properties compared to the two-component system (PAAm-silicate), additional experiments are required to understand and optimize the properties of these system for desired applications.

In a similar effort, researchers had fabricated multicomponent nanocomposite hydrogels from ferritin (iron core decorated with protein shell) and poly(vinyl alcohol) (PVA) using electrospinning (Shin et al., 2009). When subjected to aqueous media, the electrospun fibrous mesh resulted in the formation of nanocomposite hydrogels due to the swelling of PVA. The addition of ferritin significantly enhanced the mechanical stiffness and reduced creep during the cyclic deformation of the nanocomposite hydrogels. Such properties were attributed to the presence of ferritin nanoparticles that act as nanoscale springs within the PVA matrix. These types of hybrid hydrogel networks can potentially be used to fabricate scaffolds for tissue engineering applications, stimuli responsive matrix for drug release, or as actuators. Although, these multicomponent systems were able to provide superior properties compared to one or two-components systems, the complexity involved in optimizing the desired composition may need to be addressed in future.

Recent trends in designing advanced biomaterials are shifted toward designing stimuliresponsive nanocomposites. These biomaterials show significant change in their physical, chemical, or biological properties with environmental stimuli. For example, poly(Nisopropylacrylamide) (PNI-PAAm) based nanoparticles/hydrogels were used to design therapeutics device for tissue engineering and drug delivery applications. PNIPAAm exhibits a negative swelling transition at $34^{\circ} \mathrm{C}$, which makes it an attractive system from a physiological standpoint for applications in drug delivery. Such polymeric systems were further decorated with appropriate nanoparticles to develop stimuli responsive matrices. The type of nanoparticles embedded within the hydrogel networks determines the type of stimuli that can be used to change their property. A range of stimuli responsive elements such as mechanically adaptive, $\mathrm{pH} /$ enzyme/ion responsive, electrically stimulating, thermo responsive, and magnetic responsive can be incorporated within nanocomposite hydrogels. These types of responsive nanocomposite hydrogels will direct the development of next generation of nanocomposite hydrogels (Schexnailder and Schmidt, 2009; Stuart et al., 2010). Thus, there is tremendous opportunity to explore the potential of stimuli responsive nanocomposite hydrogels for biomedical and pharmaceuticals applications.

In a recent effort, Au nanoparticles were entrapped within stimuli responsive matrix to design therapeutic hydrogels (Owens et al., 2007). The researchers designed such hybrid system by functionalizing Au-nanoparticles with PEG chains, and then encapsulated these hybrid nanoparticles within thermally responsive polyacrylamide (PAAm)/poly(acrylic acid) 
(PAA) interpenetrating polymer network. Au nanoparticles have the ability to absorb visible-to-near infrared (530-1,200 nm) wavelength and thus can be used to generate heat locally. The local heating by the nanoparticles was used to trigger swelling/dwelling of the polymeric network and can resulted in the release of entrapped macromolecules. The covalently crosslinked PAAm-PAA interpenetrating polymer network can be used to deliver therapeutics using external trigger for range of biomedical and drug delivery applications.

In future, it is expected that hybrid materials will be merged with other types of technologies such as microfabrication approaches to understand cell-nanomaterials interactions.

Microscale technologies are emerging as one of the powerful technology to address some of the challenges in tissue engineering (Khademhosseini and Langer, 2007; Khademhosseini et al., 2006). For example, one of the major challenges in tissue engineering is our inability to engineer tissues with spatiotemporal control of cells, molecular cues and biophysical signals. Various microfabrication approaches are currently employed to control the cellular microenvironment. The development of photocrosslinked nanocomposite hydrogels provides an additional tool to control cellular behavior. Recent effort on GelMA-CNTs (Shin et al., 2013) and hyperbranched polyester (Zhang et al., 2013) nanocomposite hydrogels have shown that microscale technologies can be used to entrap cells in predefined geometries and cellular microenvironment. These microfabrication technologies allow better positioning of different types of cells within the hydrogel network to facilitate the formation of functional tissues. The use of microscale technologies in nanomaterials research will enable us to understand and address some of the complexity associated with engineering functional tissues.

\section{Conclusions}

Nanocomposite hydrogels are advanced biomaterials that can potentially be used for various biomedical and pharmaceutical applications. The application of these hydrogel networks includes sensors, actuators, drug delivery, stem cell engineering, regenerative medicine, and other biomedical devices. Compared to conventional polymeric hydrogels, nanocomposite hydrogels have superior physical, chemical, electrical, and biological properties. Electrically conductive scaffolds, mechanically stiff and highly elastomeric network, cell and tissue adhesive matrices, injectable matrices, and controlled drug delivery depots are some examples of nanocomposite hydrogels. The improved performance of the nanocomposite hydrogel network is mainly attributed to the enhanced interactions between the polymer chains and nanoparticles.

Designing the next generation of nanocomposite hydrogels will not only require close control over the physical, chemical, and electrical properties, but also the need to integrate suitable biological clues within the network. The synthesis and fabrication of such hydrogels network will focus on designing multicomponents network, as two-components systems are not able to incorporate multiple functionalities. Additionally, future studies of nanocomposite hydrogels will also focus on understanding the interactions between polymeric chains and nanoparticles at different length scale. This will tailor the properties of the nanocomposite hydrogels for required applications. Moreover, new fabrication technologies will also be devised to recapitulate cellular microenvironment of native tissues within the nanocomposite hydrogels.

\section{References}

Annabi N, Tamayol A, Uquillas JA, Akbari M, Bertassoni LE, Cha C, Camci-Unal G, Dokmeci MR, Peppas NA, Khademhosseini A. 25th anniversary article: Rational design and applications of hydrogels in regenerative medicine. Adv Mater. 201410.1002/adma.201303233 
Balazs AC, Emrick T, Russell TP. Nanoparticle polymer composites: Where two small worlds meet. Science. 2006; 314(5802):1107-1110. [PubMed: 17110567]

Bordes P, Pollet E, Avérous L. Nanobiocomposites: Biodegradable polyester/nanoclay systems. Prog Polym Sci. 2009; 34(2):125-155.

Caruso F. Nanoengineering of particle surfaces. Adv Mater. 2001; 13(1):11-22.

Cha C, Shin SR, Annabi N, Dokmeci MR, Khademhosseini A. Carbon-based nanomaterials: Multifunctional materials for biomedical engineering. ACS Nano. 2013; 7(4):2891-2897. [PubMed: 23560817]

Dawson JI, Oreffo ROC. Clay: New opportunities for tissue regeneration and biomaterial design. Adv Mater. 2013; 25(30):4069-4086. [PubMed: 23722321]

Discher DE, Mooney DJ, Zandstra PW. Growth factors, matrices, and forces combine and control stem cells. Science. 2009; 324(5935):1673-1677. [PubMed: 19556500]

Dvir T, Timko BP, Brigham MD, Naik SR, Karajanagi SS, Levy O, Jin H, Parker KK, Langer R, Kohane DS. Nanowired three-dimensional cardiac patches. Nat Nanotechnol. 2011; 6(11):720-725. [PubMed: 21946708]

Fisher OZ, Khademhosseini A, Langer R, Peppas NA. Bioinspired materials for controlling stem cell fate. Acc Chem Res. 2010; 43(3):419-428. [PubMed: 20043634]

Frankland S, Caglar A, Brenner D, Griebel M. Molecular simulation of the influence of chemical cross-links on the shear strength of carbon nanotube-polymer interfaces. J Phys Chem B. 2002; 106(12):3046-3048.

Gaharwar AK, Dammu SA, Canter JM, Wu C-J, Schmidt G. Highly extensible, tough, and elastomeric nanocomposite hydrogels from poly(ethylene glycol) and hydroxyapatite nanoparticles. Biomacromolecules. 2011a; 12(5):1641-1650. [PubMed: 21413708]

Gaharwar AK, Kishore V, Rivera C, Bullock W, Wu CJ, Akkus O, Schmidt G. Physically crosslinked nanocomposites from silicate-crosslinked PEO: Mechanical properties and osteogenic differentiation of human mesenchymal stem cells. Macromol Biosci. 2012; 12(6):779. [PubMed: 22517665]

Gaharwar AK, Mihaila SM, Swami A, Patel A, Sant S, Reis RL, Marques AP, Gomes ME, Khademhosseini A. Bioactive silicate nanoplatelets for osteogenic diffrentiation of human human mesenchymal stem cells. Adv Mater. 2013a; (2524):3329-3336. [PubMed: 23670944]

Gaharwar AK, Rivera C, Wu C-J, Chan BK, Schmidt G. Photo-crosslinked nanocomposite hydrogels from PEG and silica nanospheres: Structural, mechanical and cell adhesion characteristics. Mater Sci Eng C. 2013b; 33(3):1800-1807.

Gaharwar AK, Rivera CP, Wu C-J, Schmidt G. Transparent, elastomeric and tough hydrogels from poly (ethylene glycol) and silicate nanoparticles. Acta Biomater. 2011b; 7(12):4139-4148. [PubMed: 21839864]

Gaharwar AK, Schexnailder PJ, Dundigalla A, White JD, Matos-Pérez CR, Cloud JL, Seifert S, Wilker JJ, Schmidt G. Highly extensible bio-nanocomposite fibers. Macromol Rapid Commun. 2011c; 32(1):50-57. [PubMed: 21432969]

Gaharwar AK, Schexnailder PJ, Kaul V, Akkus O, Zakharov D, Seifert S, Schmidt G. Highly extensible bio-nanocomposite films with direction-dependent properties. Adv Funct Mater. 2010; 20(3):429-436.

Gaharwar AK, Wong JE, Muller-Schulte D, Bahadur D, Richtering W. Magnetic nanoparticles encapsulated within a thermoresponsive polymer. J Nanosci Nanotechnol. 2009; 9(9):5355-5361. [PubMed: 19928227]

Gillies E, Frechet J. Dendrimers and dendritic polymers in drug delivery. Drug Discov Today. 2005; 10(1):35-43. [PubMed: 15676297]

Goenka S, Sant V, Sant S. Graphene-based nanomaterials for drug delivery and tissue engineering. J Control Release. 2014; 173(1):75-88. [PubMed: 24161530]

Hench LL, Polak JM. Third-generation biomedical materials. Science. 2002; 295(5557):1014-1017. [PubMed: 11834817]

Hoppe A, Güldal NS, Boccaccini AR. A review of the biological response to ionic dissolution products from bioactive glasses and glass-ceramics. Biomaterials. 2011; 32(11):2757-2774. [PubMed: 21292319] 
Joshi N, Grinstaff M. Applications of dendrimers in tissue engineering. Curr Top Med Chem. 2008; 8(14):1225-1236. [PubMed: 18855707]

Khademhosseini A, Langer R. Microengineered hydrogels for tissue engineering. Biomaterials. 2007; 28(34):5087-5092. [PubMed: 17707502]

Khademhosseini A, Langer R, Borenstein J, Vacanti JP. Microscale technologies for tissue engineering and biology. Proc Natl Acad Sci USA. 2006; 103(8):2480-2487. [PubMed: 16477028]

Khademhosseini A, Vacanti JP, Langer R. Progress in tissue engineering. Sci Am. 2009; 300(5):6471. [PubMed: 19438051]

Kloxin AM, Kloxin CJ, Bowman CN, Anseth KS. Mechanical properties of cellularly responsive hydrogels and their experimental determination. Adv Mater. 2010; 22(31):3484-3494. [PubMed: 20473984]

Kuilla T, Bhadra S, Yao D, Kim NH, Bose S, Lee JH. Recent advances in graphene based polymer composites. Prog Polym Sci. 2010; 35(11):1350-1375.

Langer R, Vacanti JP. Tissue engineering. Science. 1993; 260(5110):920-926. [PubMed: 8493529]

Li C, Mezzenga R. Functionalization of multiwalled carbon nanotubes and their $\mathrm{pH}$-responsive hydrogels with amyloid fibrils. Langmuir. 2012; 28(27):10142-10146. [PubMed: 22712696]

Liu J, Chen C, He C, Zhao J, Yang X, Wang H. Synthesis of graphene peroxide and its application in fabricating super extensible and highly resilient nanocomposite hydrogels. ACS Nano. 2012; 6(9): 8194-8202. [PubMed: 22917015]

Liu, T.; Guo, S. Polymer nanotube nanocomposites: Synthesis, properties and applications. Salem (MA, USA): Scrivener; 2010. Properties of polyurethane/carbon nanotube nanocomposites; $\mathrm{p}$. 141-176.

Lowman, AM.; Dziubla, TD.; Bures, P.; Peppas, NA. Structural and dynamic response of neutral and intelligent networks in biomedical environments. In: Peppas, NA.; Sefton, MV., editors. Molecular and cellular foundations of biomaterials. New York: Academic Press; 2004. p. 75-130.

Lowman, AM.; Peppas, NA. Hydrogels. In: Mathiowitz, E., editor. Encyclopedia of controlled drug delivery. New York, NY: Wiley; 1999. p. 397-418.

Ma P-C, Siddiqui NA, Marom G, Kim J-K. Dispersion and functionalization of carbon nanotubes for polymer-based nanocomposites: A review. Comp A Appl Sci Manuf. 2010; 41(10):1345-1367.

Oral E, Peppas NA. Responsive and recognitive hydrogels using star polymers. J Biomed Mater Res A. 2004; 68(3):439-447. [PubMed: 14762923]

Owens DE, Eby JK, Jian Y, Peppas NA. Temperature-responsive polymer-gold nanocomposites as intelligent therapeutic systems. J Biomed Mater Res A. 2007; 83(3):692-695. [PubMed: 17530631]

Pasqui D, Atrei A, Giani G, De Cagna M, Barbucci R. Metal oxide nanoparticles as cross-linkers in polymeric hybrid hydrogels. Mater Lett. 2011; 65(2):392-395.

Peppas NA, Hilt JZ, Khademhosseini A, Langer R. Hydrogels in biology and medicine: From molecular principles to bionanotechnology. Adv Mater. 2006; 18(11):1345-1360.

Peppas, NA.; Hilt, JZ.; Thomas, JB., editors. Nanotechnology in therapeutics: Current technology and applications. Norfolk, UK: Horizon Bioscience; 2007.

Peppas NA, Huang Y, Torres-Lugo M, Ward JH, Zhang J. Physicochemical foundations and structural design of hydrogels in medicine and biology. Annu Rev Biomed Eng. 2000; 2(1):9-29. [PubMed: 11701505]

Price RL, Gutwein LG, Kaledin L, Tepper F, Webster TJ. Osteoblast function on nanophase alumina materials: Influence of chemistry, phase, and topography. J Biomed Mater Res A. 2003; 67(4): 1284-1293. [PubMed: 14624515]

Schexnailder P, Schmidt G. Nanocomposite polymer hydrogels. Colloid Polym Sci. 2009; 287(1):111.

Schexnailder PJ, Gaharwar AK II, Bartlett RL, Seal BL, Schmidt G. Tuning cell adhesion by incorporation of charged silicate nanoparticles as cross-linkers to polyethylene oxide. Macromol Biosci. 2010; 10(12):1416-1423. [PubMed: 20602416] 
Seidlits, S.; Peppas, NA. Star polymers and dendrimers in nanotechnology and drug delivery. In: Peppas, NA.; Hilt, JZ.; Thomas, JB., editors. Nanotechnology in therapeutics: Current technology and applications. 2007. p. 317-348.

Shin MK, Spinks GM, Shin SR, Kim SI, Kim SJ. Nanocomposite hydrogel with high toughness for bioactuators. Adv Mater. 2009; 21(17):1712-1715.

Shin SR, Bae H, Cha JM, Mun JY, Chen Y-C, Tekin H, Shin H, Farshchi S, Dokmeci MR, Tang S, Khademhosseini A. Carbon nanotube reinforced hybrid microgels as scaffold materials for cell encapsulation. ACS Nano. 2011; 6(1):362-372. [PubMed: 22117858]

Shin SR, Jung SM, Zalabany M, Kim K, Zorlutuna P, Kim Sb, Nikkhah M, Khabiry M, Azize M, Kong J, Wan K-t, Palacios T, Dokmeci MR, Bae H, Tang (Shirley) X, Khademhosseini A. Carbon-nanotube-embedded hydrogel sheets for engineering cardiac constructs and bioactuators. ACS Nano. 2013; 7(3):2369-2380. [PubMed: 23363247]

Skardal A, Zhang J, McCoard L, Oottamasathien S, Prestwich GD. Dynamically crosslinked gold nanoparticle-Hyaluronan hydrogels. Adv Mater. 2010; 22(42):4736-4740. [PubMed: 20730818]

Skelton S, Bostwick M, O'Connor K, Konst S, Casey S, Lee BP. Biomimetic adhesive containing nanocomposite hydrogel with enhanced materials properties. Soft Matter. 2013; 9(14):3825-3833.

Slaughter BV, Khurshid SS, Fisher OZ, Khademhosseini A, Peppas NA. Hydrogels in regenerative medicine. Adv Mater. 2009; 21(32-33):3307-3329. [PubMed: 20882499]

Sontjens SHM, Nettles DL, Carnahan MA, Setton LA, Grinstaff MW. Biodendrimer-based hydrogel scaffolds for cartilage tissue repair. Biomacromolecules. 2006; 7(1):310-316. [PubMed: 16398530]

Stuart MAC, Huck WTS, Genzer J, Müller M, Ober C, Stamm M, Sukhorukov GB, Szleifer I, Tsukruk VV, Urban M. Emerging applications of stimuli-responsive polymer materials. Nat Mater. 2010; 9(2):101-113. [PubMed: 20094081]

Thomas, J.; Peppas, N.; Sato, M.; Webster, T. Nanotechnology and biomaterials. In: Gogotsi, Y., editor. Nanomaterials handbook. Boca Raton, FL: CRC Taylor and Francis; 2006. p. 605-636.

Tibbitt MW, Anseth KS. Hydrogels as extracellular matrix mimics for 3D cell culture. Biotechnol Bioeng. 2009; 103(4):655-663. [PubMed: 19472329]

Wang E, Desai MS, Lee S-W. Light-controlled graphene-elastin composite hydrogel actuators. Nano Lett. 2013; 13(6):2826-2830. [PubMed: 23647361]

Wang Q, Mynar JL, Yoshida M, Lee E, Lee M, Okuro K, Kinbara K, Aida T. High-water-content mouldable hydrogels by mixing clay and a dendritic molecular binder. Nature. 2010; 463(7279): 339-343. [PubMed: 20090750]

Webster TJ, Siegel RW, Bizios R. Nanoceramic surface roughness enhances osteoblast and osteoclast functions for improved orthopaedic/dental implant efficacy. Scr Mater. 2001; 44(8):1639-1642.

Wu C-J, Gaharwar AK, Schexnailder PJ, Schmidt G. Development of biomedical polymer-silicate nanocomposites: A materials science perspective. Materials. 2010; 3(5):2986-3005.

Zhang H, Patel A, Gaharwar AK, Mihaila SM, Iviglia GI, Mukundan S, Bae H, Yang H, Khademhosseini A. Hyperbranched polyester hydrogels with controlled drug release and cell adhesion properties. Biomacromolecules. 2013; 14(5):1299. [PubMed: 23394067]

Zhong SP, Yung LYL. Enhanced biological stability of collagen with incorporation of PAMAM dendrimer. J Biomed Mater Res A. 2009; 91A(1):114-1122. [PubMed: 18767056] 

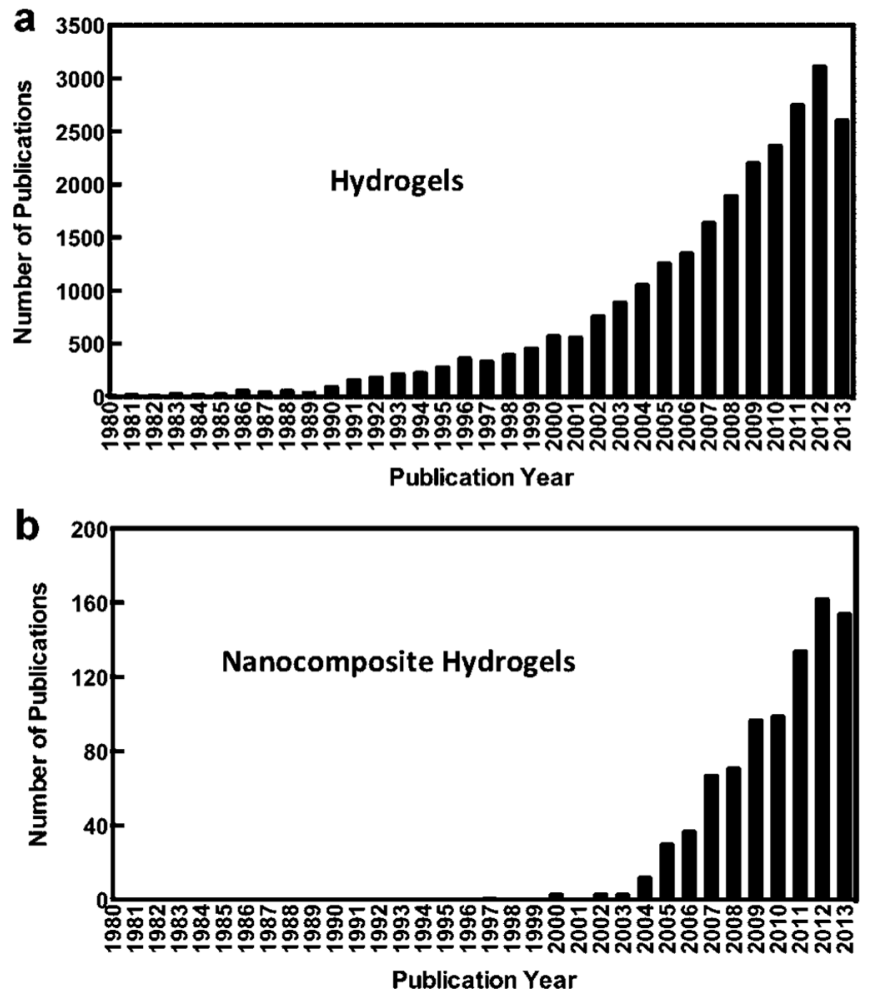

Figure 1.

Number of publications related to (a) hydrogels and (b) nanocomposite hydrogels according to ISI Web of Science (data obtained November 2013). A steady increase in the number of publication indicates growing interest in the field of nancomposite hydrogels. 
Nanocomposite Hydrogels for Biomedical Applications

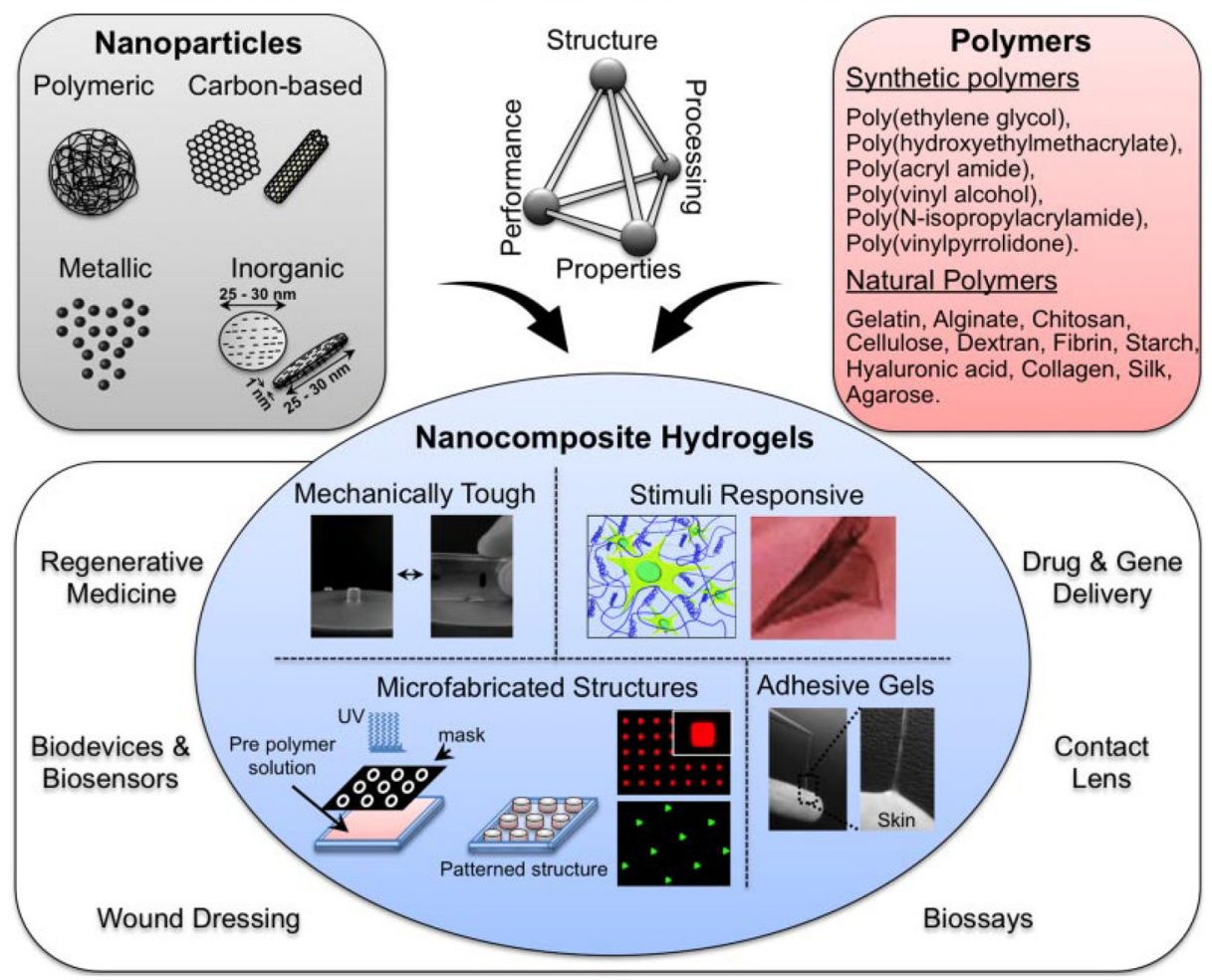

Figure 2.

Engineered nanocomposite hydrogels. A range of nanoparticles such as carbon-based nanomaterials, polymeric nanoparticles, inorganic nanoparticles, and metal/metal-oxide nanoparticles are combined with the synthetic or natural polymers to obtain nanocomposite hydrogels with desired property combinations. These nanocomposite networks are either physically or chemically crosslinked. By controlling the polymer-polymer or polymernanoparticles interactions, the physical, chemical, and biological properties of the nanocomposite hydrogels can be tailored. 
a

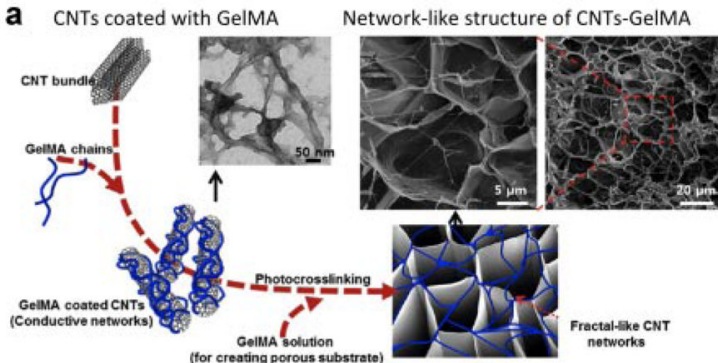

b Microfabricated Hydrogels

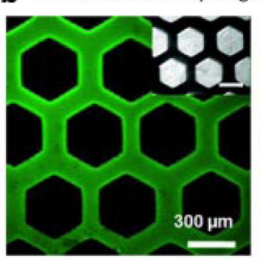

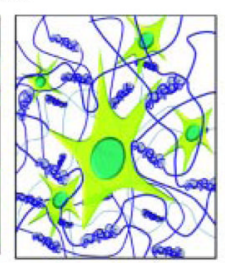

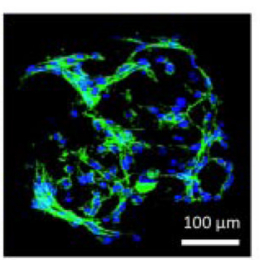

C
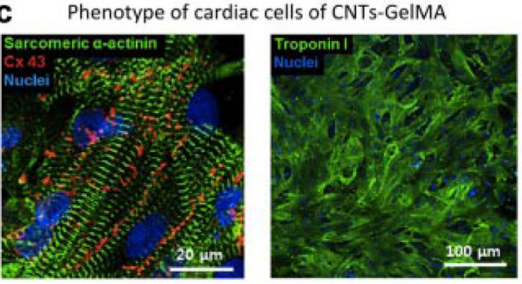

d Engineered cardiac patches from CNTs-GelMA

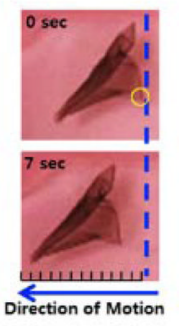

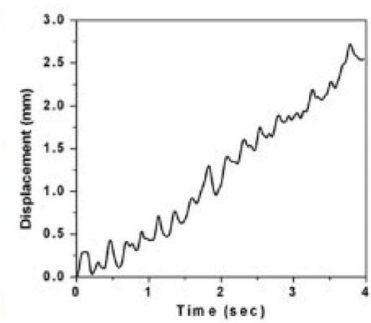

Figure 3.

Nanocomposite hydrogels from CNTs and GelMA. a: Schematic showing synthesis of nanocomposite network. First, CNTs are coated with GelMA and then the composite is subjected to UV radiation to obtain photocrosslinked network. $\mathbf{b}$ : Due to photocrosslinking ability of the nanocomposite network, microfabrication technologies can be used to control cellular interactions. c: Cardiac cells that were seeded on CNTs-GelMA nanocomposites retained their phenotype as determined by the expression of sarcomeric a-actinin and troponin I. d: The engineered cardiac patch obtained by seeding cardiac cells on CNTsGelMA surface showed macroscopic mechanical displacement due to continuous contraction and relaxation of the patch. Adapted with permission from Shin et al. (2011, 2013). Copyright (2013) American Chemical Society. 
a Hyperbranched
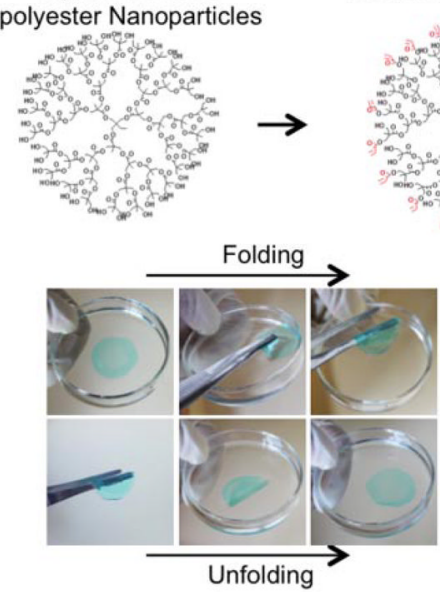

Photocrosslinkable HPE
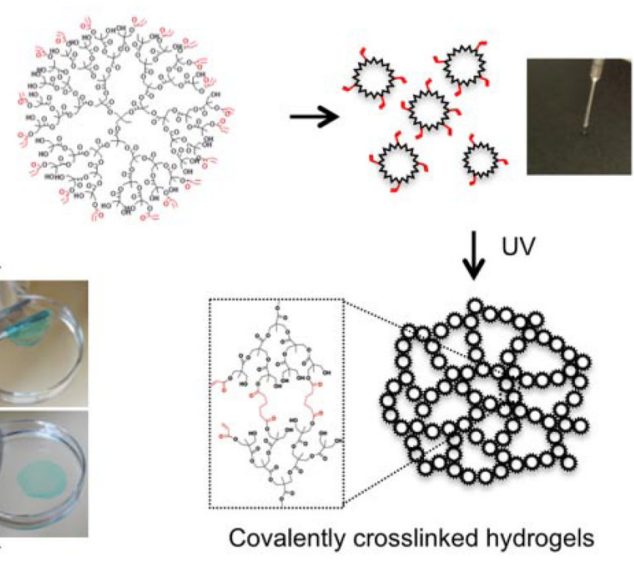

c
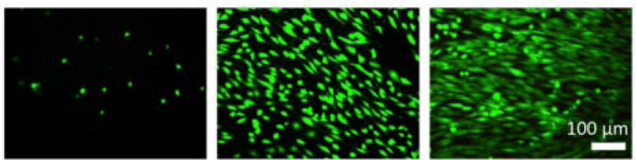

Cell $\backslash$ HPE hydrogels

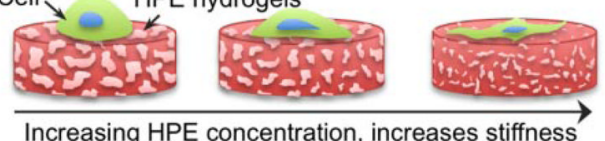

d

Microfabricated HPE Hydrogels

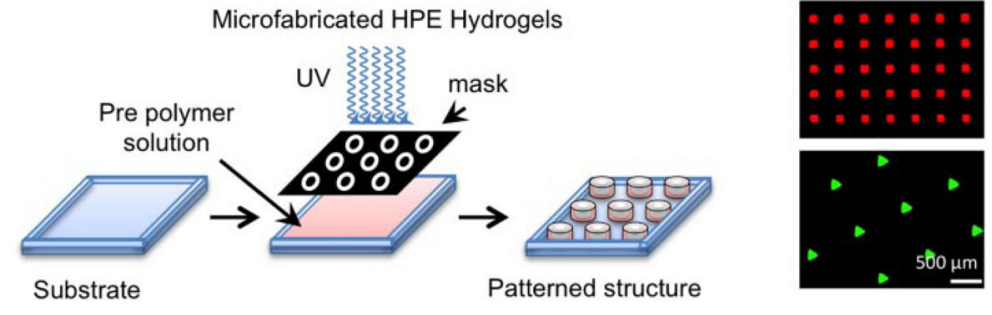

Figure 4.

Nanocomposite hydrogels from hyperbranched polyester (HPE) nanoparticles. a: HPE nanoparticles are surface functionalized with photocrosslinkable moieties. When the precursor solution containing functionalized HPE and photoinitiator is subjected to UV radiation, a covalently crosslinked network is obtained. This covalently crosslinked hydrogel is mechanically stable and can maintain its shape in physiological conditions. b: Due to the presence of hydrophobic cavities within the HPE nanoparticles, hydrophobic drugs such as dexamethasone can be entrapped. A sustain release of drug from the hydrogels network was observed over a week. c: By controlling the crosslinking density of the HPE hydrogels, physical properties and cell adhesion characteristics can be tuned. d: Photolithography technique can be used to fabricated micropatterned hydrogel structure to control the cellular interactions. Adapted with permission from Zhang et al. (2013). Copyright (2011) American Chemical Society. 
a

Precursor solution
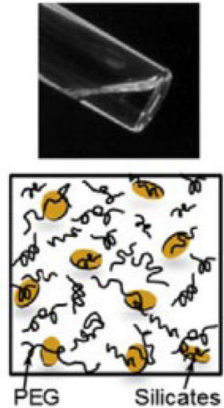

c

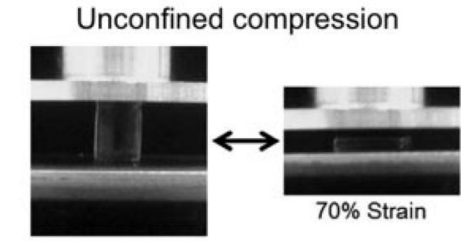

e

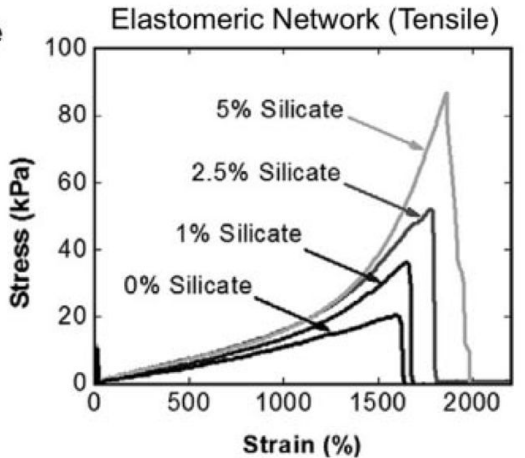

b

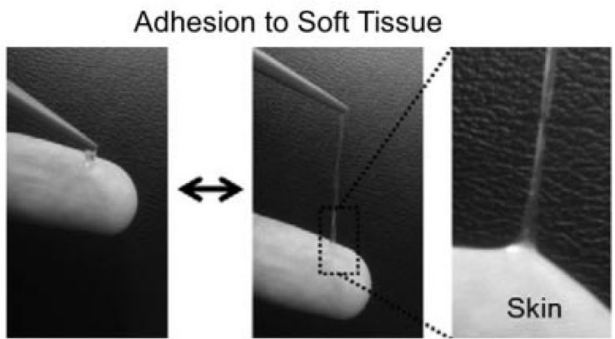

d

Mechanically Stiff Nanocomposites
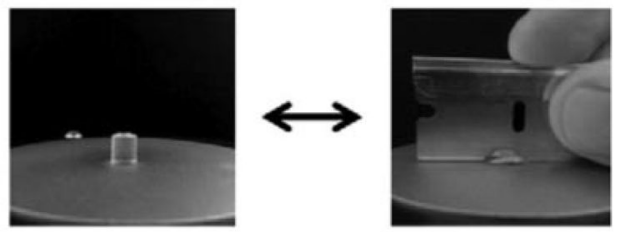

Fully hydrated

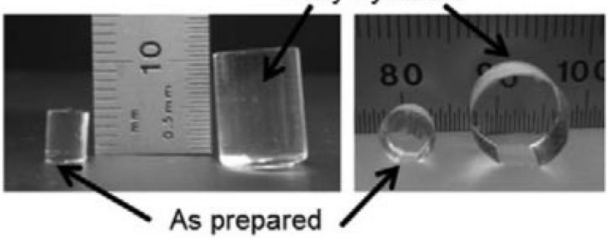

f
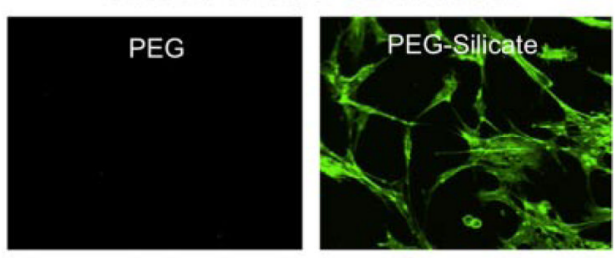

Figure 5.

Highly elastomeric hydrogel network was obtained from PEG-Silicate nanocomposites. a: Precursor solution containing silicate nanoplatelets and acrylated PEG when subjected to UV radiation results in the formation of a covalently crosslinked nanocomposite network. The nanocomposite hydrogels stick to soft tissues (b) and undergoes high deformation (c) and (d). e: The addition of synthetic silicate results in significant increase in mechanical stiffness and elongation of the nanocomposite network compared to polymeric hydrogels. f: Moreover addition of silicate also promotes cells adhesion on the nanocomposite hydrogels. Adapted with permission from Gaharwar et al. (2011b). Copyright (2013), with permission from Elsevier. 
a
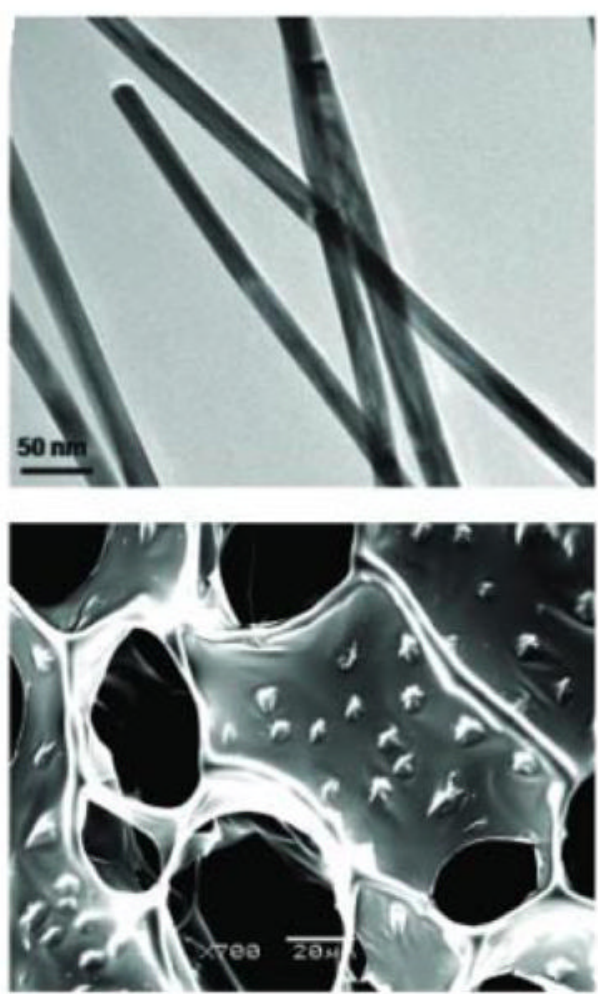

b
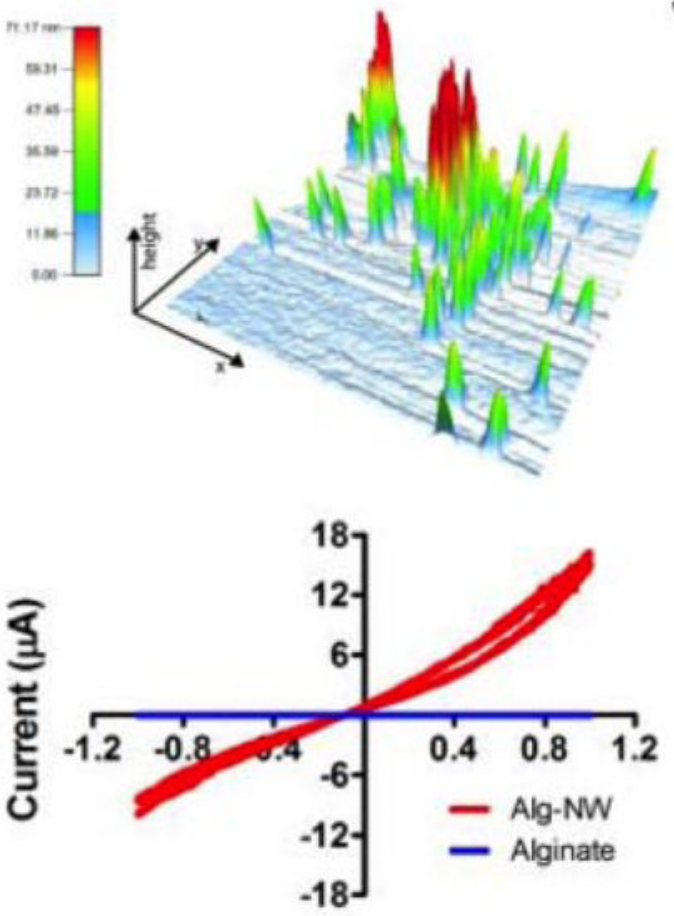

Bias (V)

c
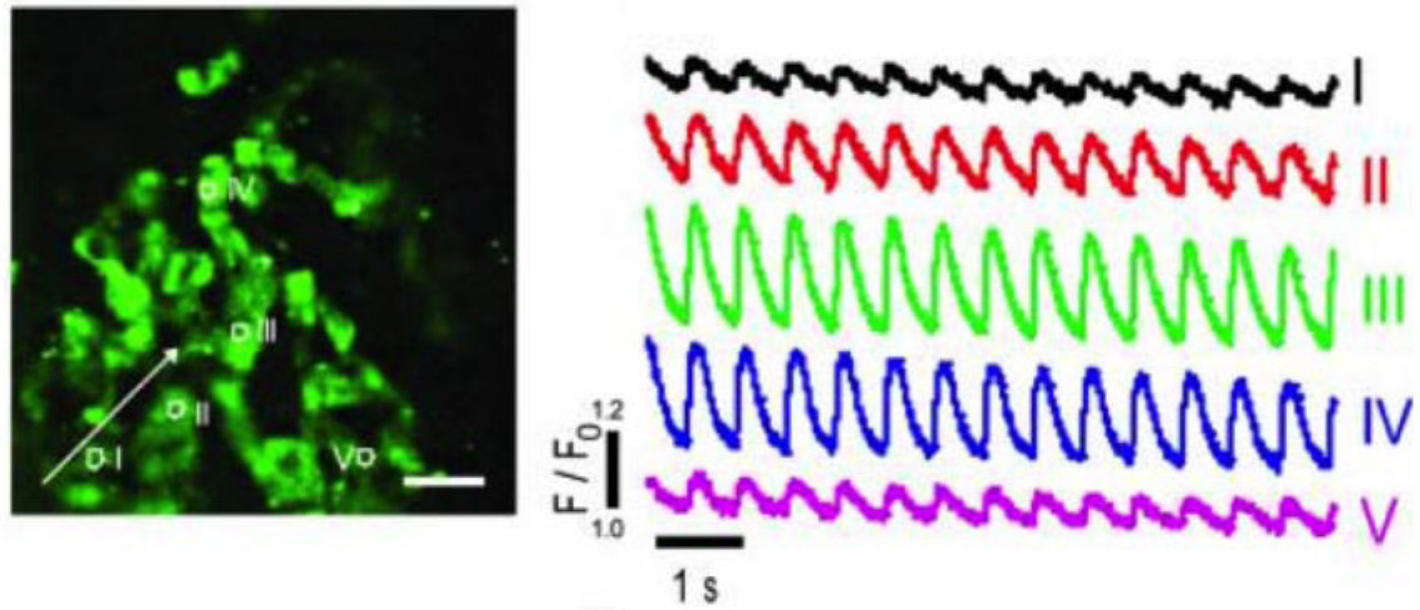

Figure 6.

Nanocomposite hydrogels containing gold nanowire and alginate. a: The distribution of gold nanowires determined using TEM indicates an average length of $\sim 1 \mu \mathrm{m}$ and an average diameter of $30 \mathrm{~nm}$. The uniform distribution of nanowire within the alginate scaffolds was also observed. b: The topographic mapping of nanocomposite detects the presence of nanowires. In nanocomposite, the current increased with bias voltage over the range -1 to $1 \mathrm{~V}$, while negligible current was passed through alginate (without nanowire) over that same range. c: Calcium transient was determined at various locations using calcium dye. The number indicates location of on the Alg-gold nanowire scaffold and the corresponding 
curves indicate calcium transport at those points. Adapted with permission from Dvir et al. (2011). Copyright (2013) Nature. 